\title{
High glucose-induced epithelial-mesenchymal transition contributes to the upregulation of fibrogenic factors in retinal pigment epithelial cells
}

\author{
DI CHE ${ }^{1 *}$, TI ZHOU ${ }^{2 *}$, YUQING LAN ${ }^{3}$, JINYE XIE $^{2}$, HAIJUN GONG $^{3}, \mathrm{CHAOYANG} \mathrm{LI}^{4}, \mathrm{JUAN}_{\mathrm{FENG}}{ }^{2}$, \\ HONGHAI HONG ${ }^{2}$, WEIWEI QI ${ }^{2}$, CAIQI MA ${ }^{2}$, QIYUAN WU ${ }^{5}$, XIA YANG ${ }^{1,2,6}$ and GUOQUAN GAO ${ }^{1,2,7}$ \\ ${ }^{1}$ Program of Molecular Medicine, Affiliated Guangzhou Women and Children's Hospital, Zhongshan School of Medicine, \\ Sun Yat-sen University; ${ }^{2}$ Department of Biochemistry, Zhongshan School of Medicine, Sun Yat-sen University; \\ ${ }^{3}$ Department of Ophthalmology, Second Affiliated Hospital, Sun Yat-sen University; ${ }^{4}$ State Key Laboratory of Ophthalmology, \\ Zhongshan Ophthalmic Center, Sun Yat-sen University; ${ }^{5}$ International Department, The Affiliated High School of \\ South China Normal University; ${ }^{6}$ Key Laboratory of Functional Molecules from Marine Microorganisms \\ (Sun Yat-sen University), Department of Education of Guangdong Province; ${ }^{7}$ China Key Laboratory of \\ Tropical Disease Control (Sun Yat-sen University), Ministry of Education, Guangzhou, Guangdong, P.R. China
}

Received April 7, 2016; Accepted September 29, 2016

DOI: $10.3892 /$ ijmm.2016.2768

\begin{abstract}
It has been reported that epithelial-mesenchymal transition (EMT) mediates multiple physiological and pathological processes. However, the occurrence and the pathogenic role of high glucose-induced EMT in retinal pigment epithelial cells (RPE cells) is unknown. The aim of this study was to examine the effects of high glucose on EMT in RPE cells. Cultured RPE cells were exposed to $25 \mathrm{mM}$ D-glucose. A vector encoding the Snail gene and siRNA targeting Snail (Snail siRNA) were transfected into the cells to induce the overexpression or silencing of Snail, respectively. AKT and extracellular signal-regulated kinase (ERK) inhibitors were used to block the activation of AKT and ERK, respectively. The levels of EMT markers, fibrogenic factors, phosphorylated ERK and phosphorylated AKT were determined by western blot analysis and immunofluorescence staining. Cell migration was evaluated by wound healing assay. Our results revealed that high glucose elevated the expression of the key EMT transcriptional factor, Snail, and that of other mesenchymal makers, and promoted cell migration. Moreover, the overexpression of Snail elevated the levels of fibronectin and connective tissue growth factor (CTGF), whereas the silencing of Snail decreased the
\end{abstract}

Correspondence to: Professor Guoquan Gao or Professor Xia Yang, Department of Biochemistry, Zhongshan School of Medicine, Sun Yat-sen University, 74 Zhongshan Road II, Guangzhou, Guangdong 510080, P.R. China

E-mail: gaogq@mail.sysu.edu.cn

E-mail: yangxia@mail.sysu.edu.cn

${ }^{*}$ Contributed equally

Key words: epithelial-mesenchymal transition, hyperglycemia, retinal pigment epithelial, fibrosis expression of fibronectin and CTGF induced by high glucose in the cells. Mechanistically, the AKT inhibitor (AKT inhibitor IV) and ERK inhibitor (U0126) significantly decreased the expression of Snail, as well as the levels of fibronectin and CTGF which were induced by high glucose. On the whole, and to the best of our knowedge, the present study is the first to demonstrate the upregulation of mesenchymal markers in RPE cells induced by high glucose, and suggest that mesenchymal transition may be involved in the pathological processes of retinal diseases.

\section{Introduction}

Retinal pigment epithelial cells (RPE cells), which are located between the choroids and the neurosensory retina, form the outer blood-retinal barrier and play a crucial role in the pathological processes that leads to the loss of vision. RPE cells are activated by the breakdown of the outer blood-retinal barrier, and can undergo proliferation and migration and secrete extracellular matrix (ECM) molecules in vitreoretinal disorders, such as proliferative diabetic retinopathy (DR), proliferative vitreoretinopathy (PVR) and age-related macular degeneration (AMD) (1-3). RPE cells are known to contribute to inflammation and fibrosis in vitreoretinal disorders (4) and in the formation of fibrotic membranes (5).

It has been documented that epithelial-mesenchymal transition (EMT) plays a role in the fibrosis of various organs, such as the kidneys, lungs and liver (6-9). There is evidence to suggest that kidney proximal tubule epithelial cells undergo EMT to induce interstitial fibrosis in diabetic nephropathy $(6,10)$. As shown in a previous study, in diabetic nephropathy, the expression of mesenchymal proteins was detected in the kidney sections of diabetic patients, and the alterations in mesenchymal proteins in tubular epithelial cells were well correlated with the declining renal function (11). Since cells undergoing EMT will lose their normal functions and mediate fibrosis in diabetic 
nephropathy, we speculated that mesenchymal transition may be involved in the development of RPE cell-related diseases.

EMT is a multi-step morphogenetic process during which epithelial cells lose their epithelial properties and acquire mesenchymal characteristics. Static epithelial cells lose cell to cell junctions, and consequently they lose apico-basal polarity to become migratory mesenchymal-like cells $(12,13)$. EMT occurs in three different biological settings with very different functional consequences (14). Type 1 EMT is invloved in original embryonic development and postnatal growth $(12,15)$. Type 2 EMT participates in wound healing, tissue regeneration and organ fibrosis. Oncogenic (type 3) EMT enables epithelial cells to acquire invasive mesenchymal phenotype characteristics that are essential in the metastatic spread (16). The most characterized transcription factors in the regulation of EMT are Snail, Slug, Twist, zinc finger E-box-binding homeobox (ZEB)1 and ZEB2 $(12,14)$.

EMT is triggered by inflammatory cytokines, cytotoxic stress and DNA damage in tissue repair and tissue fibrosis $(17,18)$. Abundant evidence indicates that hyperglycemia is etiologically related to human aging and diseases, including DR and AMD (19), and high glucose is a predictor of progression to late AMD (20). Therefore, the aim of this study was to examine the effects of high glucose on EMT in RPE cells and to determine its pathogenic role.

\section{Materials and methods}

Materials and antibodies. L-glucose and D-glucose were purchased from Sigma (St. Louis, MO, USA). AKT inhibitor IV and the extracellular signal-regulated kinase (ERK) inhibitor, U0126, were obtained from Millipore (Billerica, MA, USA) and Selleckchem (Houston, TX, USA) respectively. Antibodies to $\alpha$-smooth muscle actin ( $\alpha$-SMA; A2547) and $\beta$-actin (A5441) were purchased from Sigma-Aldrich. The antibody against phosphorylated (p-)ERK (sc-7383) was from Santa Cruz Biotechnology, Inc. (Santa Cruz, CA, USA). Antibodies against E-cadherin (610181), vimentin (550513), N-cadherin (610920) and fibronectin (610077) were obtained from BD Biosciences (Franklin Lakes, NJ, USA). Antibodies against Snail (3879S), $\beta$-catenin (9582S) and p-AKT (4060S) were from Cell Signaling Technology (Danvers, MA, USA). The antibody against connective tissue growth factor (CTGF; ab6992) was purchased from Abcam (Cambridge, MA, USA). ZO1 antibody (40-2200) was obtained from Invitrogen Life Technologies, Carlsbad, CA, USA. Goat anti-mouse (PI-2000) or anti-rabbit (PI-1000) horseradish peroxidase (HRP)-labeled secondary antibodies were from Vector Laboratories (Burlingame, CA, USA). Alexa Fluor 488 goat anti-rabbit/anti-mouse (A21206/ A21202), Alexa Fluor 594 goat anti-rabbit/anti-mouse (A21207/ A21203) antibodies and 4',6-diamidino-2-phenylindole (DAPI; D1306) were from Life Technologies (St. Louis, MO, USA).

Cell culture. ARPE19, a cell line derived from human retinal pigment epithelium (RPE) was obtained from ATCC (Manassas, VA, USA) and cultured in Dulbecco's modified Eagle's medium (DMEM) containing 10\% heat inactivated fetal bovine serum (FBS) and $100 \mathrm{U} / \mathrm{ml}$ penicillin/ streptomycin (Invitrogen Life Technologies). The cells were maintained at $37^{\circ} \mathrm{C}$ in a humidified atmosphere with $5 \% \mathrm{CO}_{2}$.
Immunofluorescence staining. Immunofluorescence staining was performed as previously described (21). For immunocytochemistry, the cells grown in 4-well glass slide chambers to $60 \%$ confluence were exposed to $25 \mathrm{mM}$ high glucose for $48 \mathrm{~h}$. The cells were then incubated with the primary antibodies specific for vimentin, $N$-cadherin and $\alpha$-SMA at a dilution of 1:200 overnight at $4^{\circ} \mathrm{C}$. The secondary antibodies (Alexa Flour 488/Alexa Flour 594 goat anti-rabbit/anti-mouse) were then added at a dilution of 1:200 for $1 \mathrm{~h}$. Slides were prepared with a mounting medium containing DAPI to counterstain the nucleus.

Western blot analysis. The cells were lysed for total protein extraction using RIPA buffer. The protein concentration was determined using a Bio-Rad DC protein assay kit (Bio-Rad Laboratories, Hercules, CA, USA) according to the manufacturer's instructions. The aliquots of equal amounts of protein were resolved by sodium dodecyl sulfate-polyacrylamide gel electrophoresis (SDS-PAGE) and transferred onto a PVDF membrane (Bio-Rad Laboratories). After blocking with 5\% non-fat dry milk in Tris-buffered saline Tween-20 (TBST) for $1 \mathrm{~h}$, the membrane was incubated overnight at $4^{\circ} \mathrm{C}$ with various primary antibodies. After washing with TBST, the membrane was incubated with the appropriate secondary antibody for $2 \mathrm{~h}$. The membrane was again washed with TBST, and immunoblots were developed with the enhanced chemiluminescent reagents from Pierce/Thermo Fisher Scientific (Waltham, MA, USA) according to the manufacturer's instructions. Images were acquired using ImageQuant Las 4000 mini (GE Healthcare Bio-sciences, Pittsburgh, PA, USA) and densitometry was performed using ImageJ software and normalized to the $\beta$-actin levels.

Reverse transcription-quantitative PCR (RT-qPCR). Total RNA was extracted from the cultured cells using TRIzol reagent according to the manufacturer's instructions (Invitrogen Life Technologies). Total RNA (500 ng) was used for reverse transcription using the PrimeScript ${ }^{\circledR}$ RT reagent kit (perfect real-time) (Takara Bio Inc., Otsu, Japan). The cDNA was used for quantitative PCR (qPCR) using SYBR ${ }^{\circledR}$ Premix Ex Taq ${ }^{\text {TM }}$ (rerfect real-time) (Takara Bio Inc.) and a Roche capillary-based LightCycler $^{\circledR} 2.0$ system (Roche Diagnostics, Indianapolis, IN, USA). The specificity of the amplification reactions was confirmed by melting curve analysis. All expression data were normalized to those for $\beta$-actin. The data were quantified by the comparative threshold cycle $(\mathrm{Ct})$ method for relative gene expression. The PCR cycling conditions were as follows: $95^{\circ} \mathrm{C}$ for $30 \mathrm{sec}, 95^{\circ} \mathrm{C}$ for $5 \mathrm{sec}$ and $60^{\circ} \mathrm{C}$ for $45 \mathrm{sec}$ for 40 cycles. Primer sequences are as follows: human snail forward, TGCGCTACTGCTGCGCGAAT and reverse, GGGCTGCTGGAAGGTAAACTCTGGA; $\beta$-actin forward, GCACTCTTCCAGCCTTCCTT and reverse, GTTGG CGTACAGGTCTTTGC.

Wound healing assay. The cells were seeded in each well of a 6-well culture plate and then cultured for $24 \mathrm{~h}$ until they reached approximately $80 \%$ confluence. The cells were starved in DMEM for $24 \mathrm{~h}$ and then exposed to L-glucose as a control and D-glucose $(25 \mathrm{mM})$ for $48 \mathrm{~h}$. Images of the wells under a microscope (Zeiss Axio Observer Z1, Carl Zeiss Meditec AG, Jena, Germany) were acquired the indicated time points after the wound scratch was made. The migration rate of the cells was 

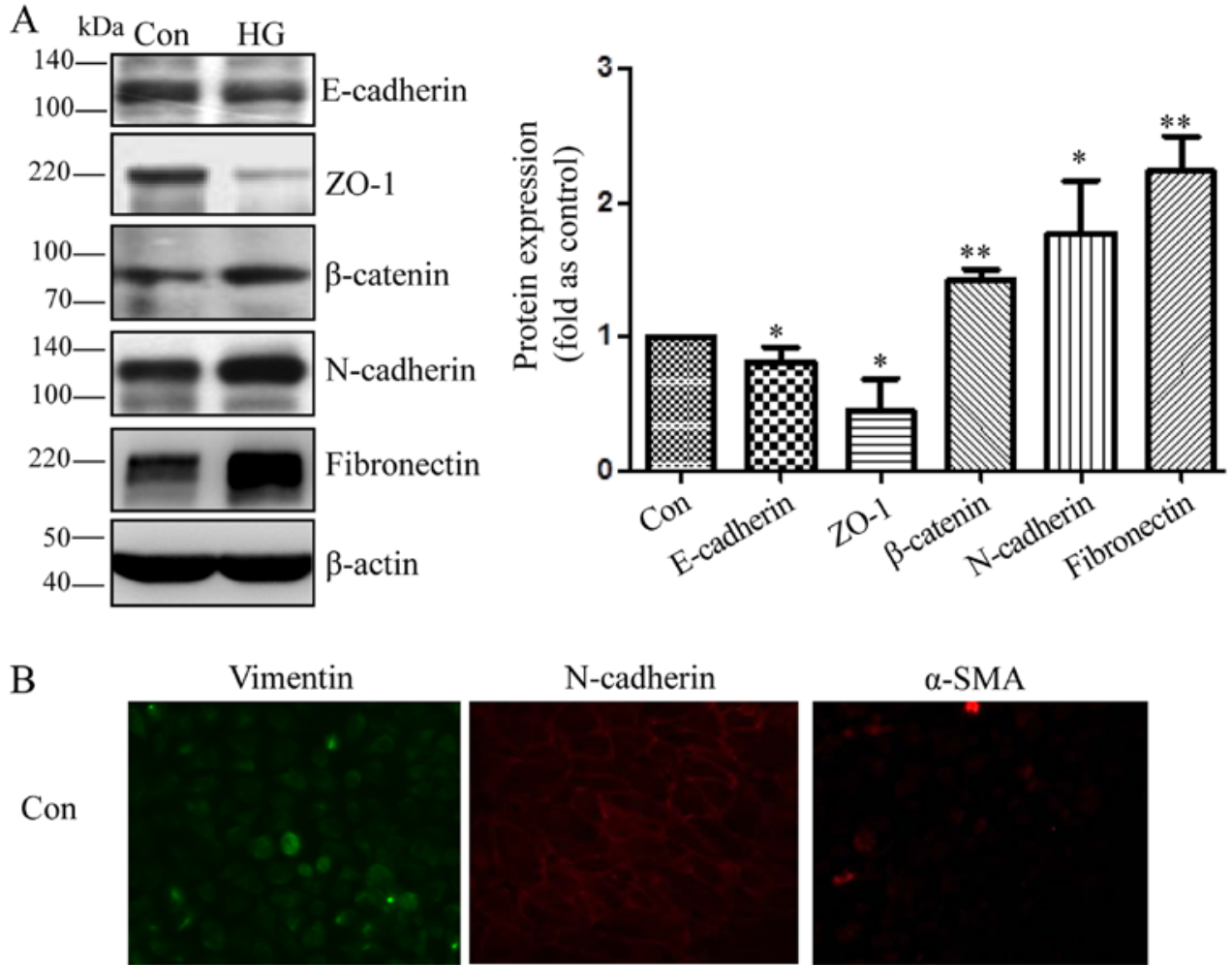

HG
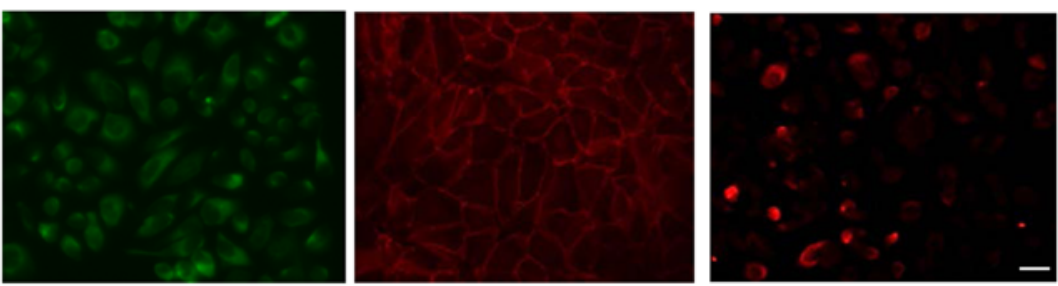

Figure 1. Mesenchymal transition of retinal pigment epithelial cells (RPE cells) induced by exposure to high glucose. (A) Representative western blots of E-cadherin, Zonula occludens-1 (ZO-1), $\beta$-catenin, $\mathrm{N}$-cadherin, fibronectin in the cells exposed to $25 \mathrm{mM}$ D-glucose (HG) and L-glucose as an osmotic control for the indicated periods of time $\left(48 \mathrm{~h}\right.$ ). The bands were quantified relative to $\beta$-actin (means $\pm \mathrm{SD},{ }^{* *} \mathrm{p}<0.01,{ }^{*} \mathrm{p}<0.05, \mathrm{n}=3$ ). (B) RPE cells were grown on glass coverslips for $24 \mathrm{~h}$, starved for $24 \mathrm{~h}$, and then incubated with $25 \mathrm{mM} \mathrm{D}$-glucose (HG) and L-glucose as a control for $48 \mathrm{~h}$. Compared with the control cells, the HG-exposed cells displayed an increased expression of vimentin, $\mathrm{N}$-cadherin and $\alpha$-SMA, as shown by immunofluorescence staining. Original magnification, $\mathrm{x} 400$.

calculated as the distance traveled by the cells from the wound edge to the cell-free space.

RNA interference. Oligonucleotides matching the selected regions of human Snail and scrambled siRNAs that were used as a negative control were purchased from RiboBio (Guangzhou, China). The cells were transfected with siRNA oligonucleotides at a final concentration of $100 \mathrm{nM}$ with HiPerFect (Qiagen, Carson City, CA, USA) according to the manufacturer's instructions. The cells were transfected with siRNA oligonucleotides for $24 \mathrm{~h}$ then followed by incubation in the presence of high glucose for an additional $48 \mathrm{~h}$.

Cell transfection with overexpression vector. Full-length Snail cDNA was a gift from Professor Jun Li (Sun Yat-sen University, Guangzhou, China). The pCR3.1-vector and pCR3.1-Snail plasmid were transfected into the cells using Lipofectamine 2000 according to the manufacturer's instructions (Invitrogen Life Technologies). After $48 \mathrm{~h}$, the cells were harvested, and the expression of proteins was determined using western blot analysis.
Statistical analysis. Data are presented as the means \pm SD. Comparisons were performed by a two-tailed paired Student's t-test. A value of $\mathrm{p}<0.05$ was considered to indicate a statistically significant difference.

\section{Results}

EMT is induced by high glucose in RPE cells. RPE cells are the key component of the outer blood-retina barrier and the main contributor to the development of fibrotic tissue in the retina (22). Therefore, we evaluated the direct effect of high glucose on mesenchymal transition in RPE cells. As shown in Fig. 1, compared to exposure to L-glucose as an osmotic control, exposure to $25 \mathrm{mM}$ high glucose for $48 \mathrm{~h}$ elevated the levels of $\mathrm{N}$-cadherin, $\beta$-catenin, fibronectin and decreased the levels of E-cadherin and ZO-1 in the RPE cells (Fig. 1A). Moreover, immunofluorescence staining revealed that the cells exposed to high glucose had more intensive vimentin, $\mathrm{N}$-cadherin and $\alpha$-SMA signals compared with the control cells (Fig. 1B).

It is well known that EMT can increase cell motility (23-25). Normal RPE cells are quiescent without migration $(26,27)$. In 

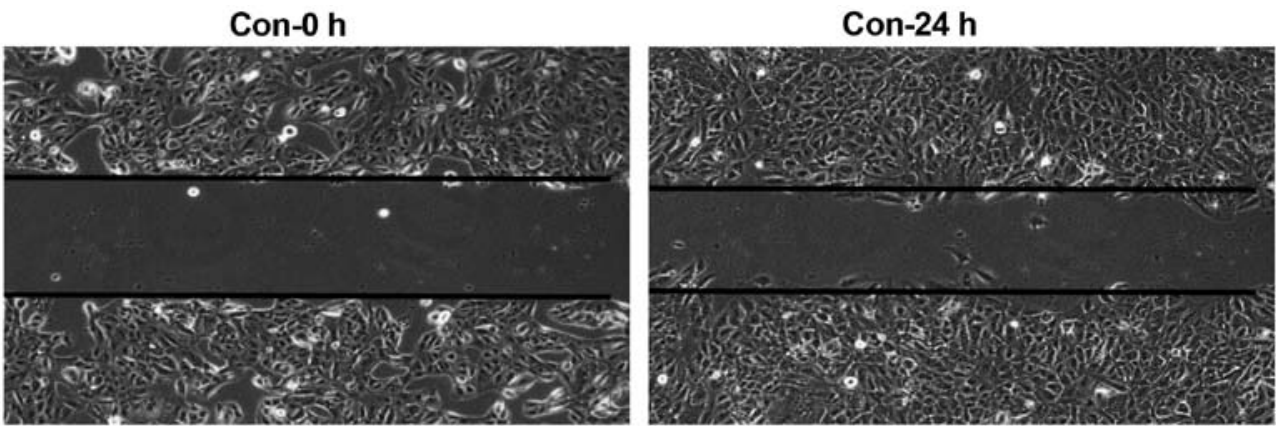

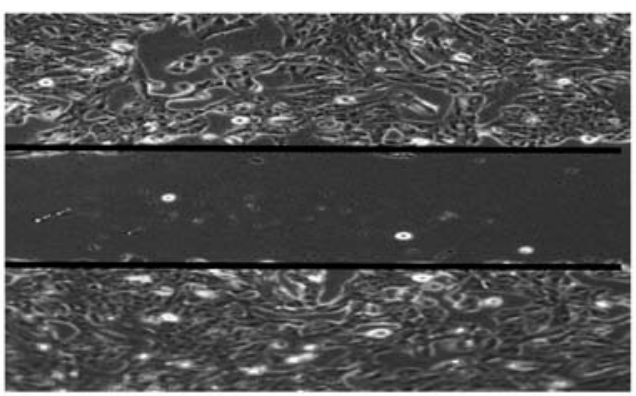

HG-0 $h$

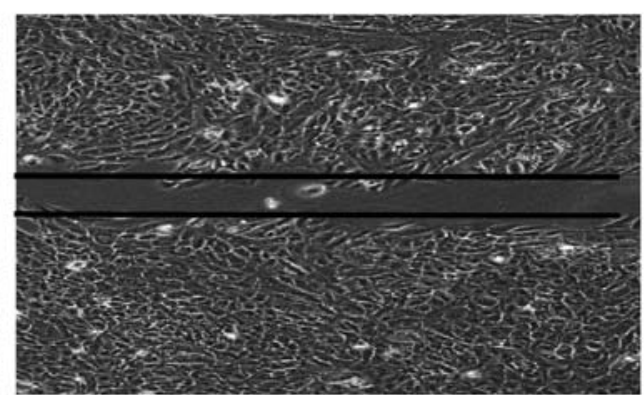

HG-24 h

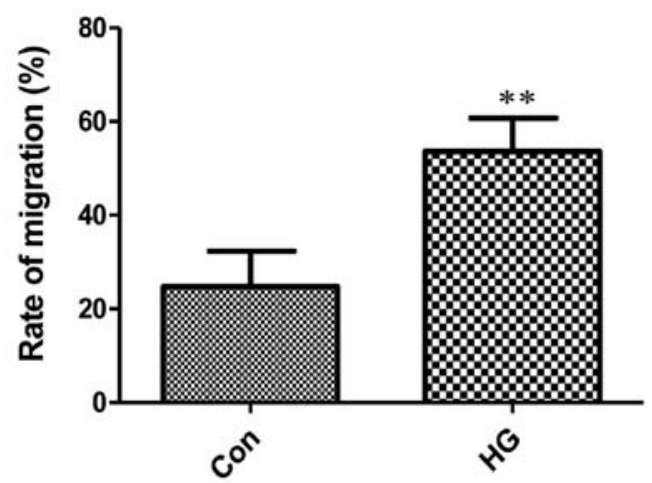

Figure 2. Exposure to high glucose HG) promotes the migration of retinal pigment epithelial cells (RPE cells). ARPE19 cells were grown as a monolayer in 6 -well plates until approximately $80 \%$ confluence. Cells were starved in DMEM for $24 \mathrm{~h}$ and then exposed to L-glucose and D-glucose ( $25 \mathrm{mM}$ ) for $48 \mathrm{~h}$. The migration rate was measured by the migration distance after a scratch was made. These experiments were conducted independently in triplicate. The data are presented as the means $\pm \mathrm{SD},{ }^{* *} \mathrm{p}<0.01$ compared with L-glucose as the control.

this study, the number of migrated cells in the high glucoseexposed RPE cells was considerably higher than the mean number of migrated control cells (Fig. 2). These observations indicated that the RPE cells exposed to high glucose underwent mesenchymal transition and migration was initiated.

Exposure to high glucose induces the upregulation of Snail. Snail is a classical transcription factor involved in EMT in tumors (14). Therefore, in this study, we investigated whether the expression of Snail was upregulated by exposure of the cells to high glucose. We found that compared to the control cells, high glucose increased the mRNA expression of Snail $24 \mathrm{~h}$ following exposure and reached the highest level at $48 \mathrm{~h}$ (Fig. 3A). Likewise, the promoting effect of high glucose on the Snail protein level at $48 \mathrm{~h}$ was also confirmed by western blot analysis (Fig. 3B).

Snail promotes the expression of cytokines in RPE cells. CTGF and fibronectin are important profibrotic growth factors that induce the production of ECM components and angiogenesis $(28,29)$. CTGF and fibronectin have been implicated in the pathological progress in patients with vitreoretinal disorders (30-34) and is induced by high glucose $(31,35)$. It has been reported that EMT is associated with fibrogenesis in diabetic nephropathy and other organs (6-9). In order to fully understand the pathogenic role of mesenchymal transition in RPE cells, we transfected the cells with Snail expression vector. Our results revealed that the protein level of Snail was upregulated in the cells transfected with the Snail overexpression vector compared to the cells transfected with the empty vector (Fig. 4A). In addition, with the overexpression of Snail, the expression of fibronectin and CTGF also increased in RPE cells, as shown by western blot analysis (Fig. 4A). Furthermore, compared to controls transfected with scrambled siRNA, the silencing of Snail decreased expression of CTGF and fibronectin in RPE cells, which had been increased by high glucose (Fig. 4B). These data suggested that mesenchymal transition in RPE cells may contribute to fibrosis by promoting the secretion of important cytokines.

The AKT and ERK signaling pathways mediate the expression of mesenchymal markers induced by high glucose in RPE cells. 

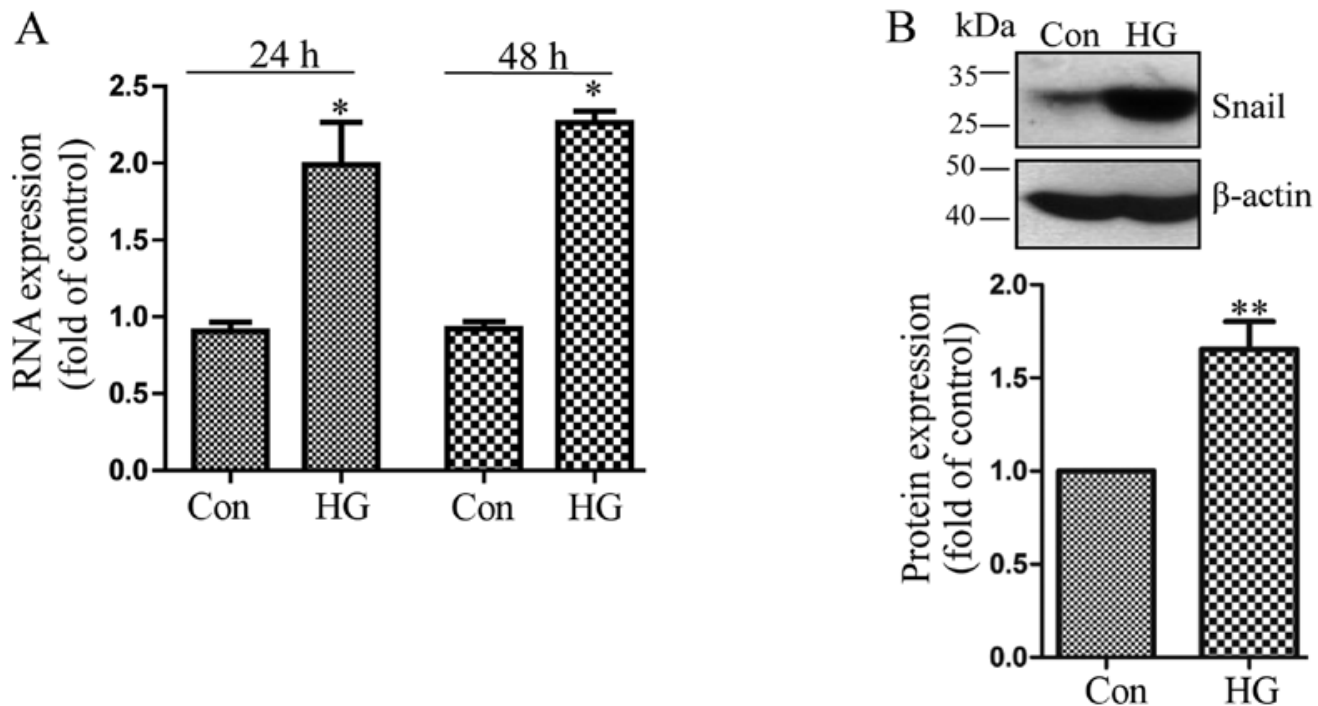

Figure 3. Snail was upregulated by exposure to high glucose. (A) RT-qPCR and (B) western blot analysis of Snail expression in the cells exposed to $25 \mathrm{mM}$ D-glucose (HG) and L-glucose as a control for indicated periods of time.
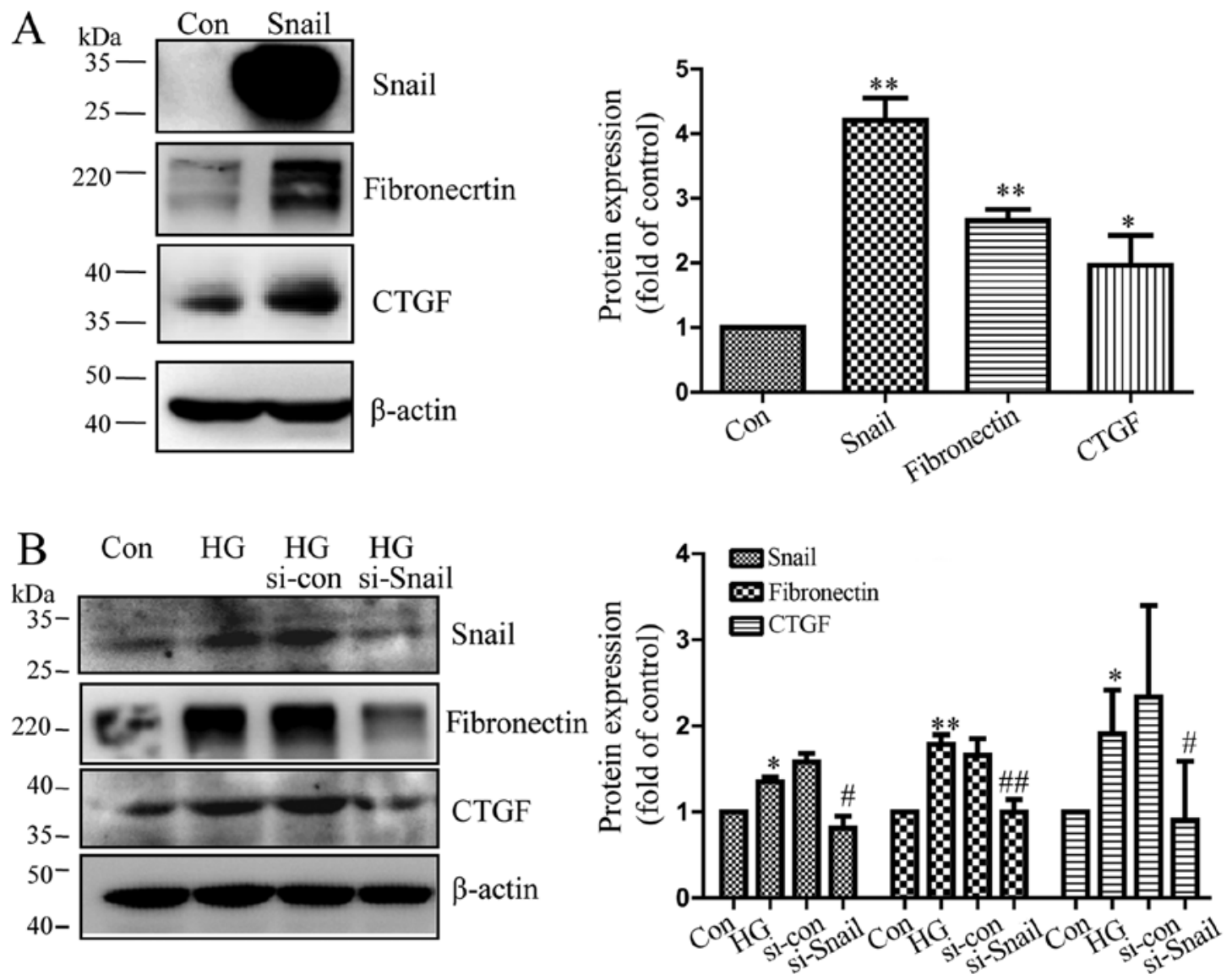

Figure 4. Snail regulates the expression of connective tissue growth factor (CTGF)/fibronectin in retinal cells. (A) Retinal pigment epithelial cells (RPE cells) were transfected with pCR3.1-mock plasmid and pCR3.1-Snail plasmid for $48 \mathrm{~h}$. (B) RPE cells were transfected with control siRNA or Snail siRNA for $24 \mathrm{~h}$ followed by incubation in the presence of high glucose for an additional $48 \mathrm{~h}$. Total cellular proteins were immunoblotted with antibodies to fibronectin and CTGF. Blots are representatives of 3 independent experiments. The bands were quantified relative to $\beta$-actin (means $\pm \mathrm{SD},{ }^{* *} \mathrm{p}<0.01$ and ${ }^{*} \mathrm{p}<0.05, \mathrm{n}=3$ ).

The activation AKT and ERK plays a critical role in the process of epithelial-mesenchymal transition (36-38). The AKT and ERK pathways have been recently recognized as new players in retinal disorders (39-41). These findings led us to hypothesize that the AKT and ERK signaling pathways regulate the expression of mesenchymal markers in RPE cells. To confirm 

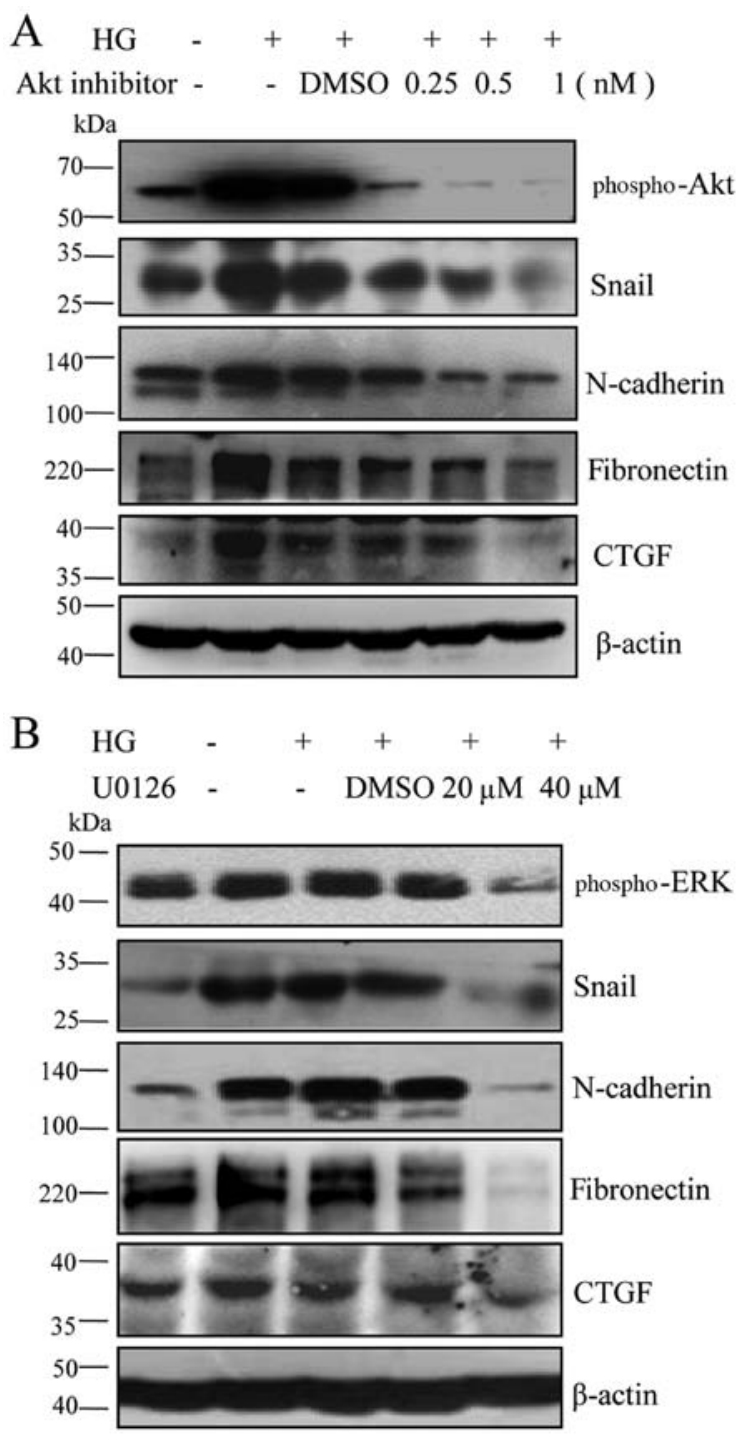

Figure 5. The AKT and ERK signaling pathways mediate the expression of Snail/N-cadherin induced by high glucose. Retinal pigment epithelial cells (RPE cells) were pre-treated for $2 \mathrm{~h}$ with (A) the Akt, Akt inhibitor IV and (B) the ERK inhibitor, U0126. The inhibitors were washed out, and the RPE cells were stimulated with $25 \mathrm{mM}$ D-glucose (HG) and L-glucose as a control for $48 \mathrm{~h}$. Gel shows a representative experiment from 3 independent determinations.

the hypothesis, we used the AKT, AKT inhibitor IV and the ERK inhibitor, U0126, to block these signaling pathways. Our results revealed that AKT inhibitor IV and U0126 downregulated the levels of Snail and N-cadherin in a dose-dependent manner, followed by a decrease in the levels of CTGF and fibronectin (Fig. 5). These data indicated that the AKT and ERK signaling pathways were involved in the high glucoseinduced mesenchymal transition and fibrosis in RPE cells.

\section{Discussion}

The present study reports that hyperglycemia induces mesenchymal transition in cultured RPE cells. The overexpression of Snail increased the protein levels of fibronectin and CTGF. Likewise, the silencing of Snail using siRNA decreased the expression of fibronectin and CTGF which was induced by high glucose in RPE cells. Mechanism experiments indicated that blockade of the AKT and ERK signaling pathways using chemical inhibitors decreased the expression of Snail, as well as that of fibronectin and CTGF, which had been induced by high glucose in RPE cells.

EMT is observed in the process of renal interstitial fibrosis, of pulmonary fibrosis, of liver fibrosis, or in specific ocular tissue (6-11). Most vision loss occurs following the transition from a disease of inflammation to a disease of neovascular fibrosis (42). RPE cells form the outer blood retinal barrier from the choroidal capillary bed by separating the outer retina. The dysfunction of the RPE can result in retinal edema, detachment or degeneration (43). Normal RPE cells are quiescent without proliferation or migration abilities $(26,27)$. In this study, we found that high glucose induced the expression of mesenchymal makers in RPE cells (Fig. 1). Subsequently, the activated RPE cells induced by high glucose underwent migration (Fig. 2). Moreover, it has been proposed that hyperglycemia increases superoxide production, which in turn initiates accelerated advanced glycation end-product (AGE) formation and exacerbates interrelated pathogenic responses. AGEs are one of the important factors involved in the pathogenesis of diseases of the eye, and it has been demonstrated that AGE mimetic administration induces the breakdown of RPE function in RPE cells (44). We speculated that the pathogenic role of AGE in RPE was partially ascribed to the induction of mesenchymal transition. In this study, we verified that AGE-stimulated cells displayed an altered mesenchymal morphology with a decreased expression of E-cadherin and an increased expression of vimentin by immunofluorescence staining. AGE significantly elevated the Snail mRNA level (data not shown).

Cao et al newly proved the existence endothelial to mesenchymal transition (EndMT) in diabetic retinas (45). Our data, together with their study extend our understaning of mesenchymal transition specific to diabetic retinas. These observations indicate that retinal cells in the setting of hyperglycemia undergo mesenchymal transition, and this may be the initial and key event that is responsible for cellular dysfunction and the development of vitreoretinal diseases.

In this study, we demonstrated that hyperglycemia induced the cell transition from a normal phenotype to a mesenchymal phenotype and promoted Snail expression (Fig. 3). It would be of interest to determine the consequence of this transition concerning the pathogenic progress. In this study, to the best of our knowledge, we demonstrate for the first time that Snail regulated the expression of CTGF and fibronectin, which are important fibrogenic factors produced by RPE cells (Fig. 4). Due to the location of these cells, we hypothesized that the occurrence of mesenchymal transition in RPE cells would lead to the production of cytokines that results in indirect effects on the retina. Moreover, we hypothesized that mesenchymal transition leads to the cellular dysfunction partly through abnormal cytokine secretion and may participate in the functions of retinal cells, namely their fuctions other than fibrosis, such as intraretinal micovasular abnormalities.

This point warrants further investigation. Furthermore, recent studies have reported that CTGF itself induces EMT in renal cells $(46,47)$. If this is also the case in retinal cells, we can assume that CTGF and Snail form a positive loop, resulting in a vicious circle of the development of vitreoretinal disorders. 
Transforming growth factor (TGF)- $\beta$ has been shown to play a central role in initiating EMT, and has been extensively studied. Therefore, we expected to elucidate a novel mechanism other than TGF- $\beta$, which could modulate mesenchymal transition in RPE cells. Recent data indicate that the normal epithelial phenotype and cell proliferation and migration appear to be associated with the activation of AKT and ERK via their phosphorylation $(36,38)$. The connection between AKT and Snail and cell-cell adhesion plays a role in various tumors, as well as in the repair of normal tissue after wounding (48). Moreover, the Ras-ERK pathway is required for EMT, and it cooperates with other pathways to upregulate the expression of EMT-related genes, including mesenchymal genes and transcriptional repressors (e.g. Snail, Slug, Twist and ZEB) (37). However, these studies were confined to EMT in tumors. It is unknown as to which signaling pathways are involved in mesenchymal transition in RPE cells. In this study, we found that high glucose induced AKT and ERK phosphorylation followed by the induction of Snail and $\mathrm{N}$-cadherin expression, as well as that of fibrogenic factors, while the blockade of the signaling pathways decreased the expression of Snail, N-cadherin, fibronectin and CTGF (Fig. 5). These findings indicated a novel mechanism through which the AKT and ERK signaling pathways modulate RPE dysfunction, relying on the regulation of mesenchymal transition, and that the signaling pathways may cooperate with each other.

In conclusion, the findings of our study, to the best of our knowledge, demonstrate for the first time that high glucose induces mesenchymal transition in RPE cells and suggest that the AKT and ERK signaling pathways regulate the expression of mesenchymal markers in RPE cells.

\section{Acknowledgements}

This study was supported by the National Nature Science Foundation of China, grant nos. 81200706, 81172163, 81272338, $81272515,81400639,81370945,81471033,81572342,81570871$ and 81570764; the National Key Sci-Tech Special Project of China, grant no. no. 2013ZX09102-053; the Program for Doctoral Station in University, grant nos. 20120171110053 and 20130171110053; the Fundamental Research Funds for the Central Universities' Youth Cultivation Project of China, grant no. 50000-3161046; the Guangdong Natural Science Fund, grant nos. S2012040006986, S2012010009250 and 2015A030313103; and the Key Sci-Tech Research Project of Guangzhou Municipality, China, grant nos. 2011Y1-00017-8, 12A52061519 and 201508020033.

\section{References}

1. Campochiaro PA: Pathogenic mechanisms in proliferative vitreoretinopathy. Arch Ophthalmol 115: 237-241, 1997.

2. Esser P,Heimann K,Bartz-schmidt KU,Fontana A,SchraermeyerU, Thumann $\mathrm{G}$ and Weller M: Apoptosis in proliferative vitreoretinal disorders: Possible involvement of TGF-beta-induced RPE cell apoptosis. Exp Eye Res 65: 365-378, 1997.

3. Miller H, Miller B and Ryan SJ: The role of retinal pigment epithelium in the involution of subretinal neovascularization. Invest Ophthalmol Vis Sci 27: 1644-1652, 1986.

4. Bastiaans J, van Meurs JC, van Holten-Neelen C, Nijenhuis MS, Kolijn-Couwenberg MJ, van Hagen PM, Kuijpers RWAM, Hooijkaas H and Dik WA: Factor Xa and thrombin stimulate proinflammatory and profibrotic mediator production by retinal pigment epithelial cells: A role in vitreoretinal disorders? Graefes Arch Clin Exp Ophthalmol 251: 1723-1733, 2013.
5. Qin D, Zhang GM, Xu X and Wang LY: The PI3K/Akt signaling pathway mediates the high glucose-induced expression of extracellular matrix molecules in human retinal pigment epithelial cells. J Diabetes Res 2015: 920280, 2015.

6. Carew RM, Wang B and Kantharidis P: The role of EMT in renal fibrosis. Cell Tissue Res 347: 103-116, 2012

7. Nowrin K, Sohal SS, Peterson G, Patel R and Walters EH: Epithelial-mesenchymal transition as a fundamental underlying pathogenic process in COPD airways: Fibrosis, remodeling and cancer. Expert Rev Respir Med 8: 547-559, 2014.

8. Chapman HA: Epithelial-mesenchymal interactions in pulmonary fibrosis. Annu Rev Physiol 73: 413-435, 2011.

9. Lee SJ, Kim KH and Park KK: Mechanisms of fibrogenesis in liver cirrhosis: The molecular aspects of epithelial-mesenchymal transition. World J Hepatol 6: 207-216, 2014.

10. Srivastava SP, Koya D and Kanasaki K: MicroRNAs in kidney fibrosis and diabetic nephropathy: Roles on EMT and EndMT. BioMed Res Int 2013: 125469, 2013.

11. Liu Y: New insights into epithelial-mesenchymal transition in kidney fibrosis. J Am Soc Nephrol 21: 212-222, 2010.

12. Samatov TR, Tonevitsky AG and Schumacher U: Epithelialmesenchymal transition: Focus on metastatic cascade, alternative splicing, non-coding RNAs and modulating compounds. Mol Cancer 12: 107, 2013.

13. Kalluri R and Weinberg RA: The basics of epithelial-mesenchymal transition. J Clin Invest 119: 1420-1428, 2009.

14. Zeisberg M and Neilson EG: Biomarkers for epithelial-mesenchymal transitions. J Clin Invest 119: 1429-1437, 2009.

15. Chaffer CL, Thompson EW and Williams ED: Mesenchymal to epithelial transition in development and disease. Cells Tissues Organs 185: 7-19, 2007.

16. Thiery JP: Epithelial-mesenchymal transitions in tumour progression. Nat Rev Cancer 2: 442-454, 2002.

17. Hirasawa M, Noda K, Noda S, Suzuki M, Ozawa Y, Shinoda K, Inoue M, Ogawa Y, Tsubota K and Ishida S: Transcriptional factors associated with epithelial-mesenchymal transition in choroidal neovascularization. Mol Vis 17: 1222-1230, 2011.

18. Thiery JP and Sleeman JP: Complex networks orchestrate epithelial-mesenchymal transitions. Nat Rev Mol Cell Biol 7: 131-142, 2006.

19. Chiu CJ and Taylor A: Dietary hyperglycemia, glycemic index and metabolic retinal diseases. Prog Retin Eye Res 30: 18-53, 2011.

20. Ghaem Maralani H, Tai BC, Wong TY, Tai ES, Li J, Wang JJ and Mitchell P: Metabolic syndrome and risk of age-related macular degeneration. Retina 35: 459-466, 2015.

21. Zhou T, Hu Y, Chen Y, Zhou KK, Zhang B, Gao G and Ma JX: The pathogenic role of the canonical Wnt pathway in age-related macular degeneration. Invest Ophthalmol Vis Sci 51: 4371-4379, 2010.

22. Snead DR, James S and Snead MP: Pathological changes in the vitreoretinal junction 1: Epiretinal membrane formation. Eye (Lond) 22: 1310-1317, 2008.

23. Hazan RB, Phillips GR, Qiao RF, Norton L and Aaronson SA: Exogenous expression of $\mathrm{N}$-cadherin in breast cancer cells induces cell migration, invasion, and metastasis. J Cell Biol 148: 779-790, 2000.

24. Williams E, Williams G, Gour BJ, Blaschuk OW and Doherty P: A novel family of cyclic peptide antagonists suggests that $\mathrm{N}$-cadherin specificity is determined by amino acids that flank the HAV motif. J Biol Chem 275: 4007-4012, 2000.

25. De Wever O, Westbroek W, Verloes A, Bloemen N, Bracke M, Gespach C, Bruyneel E and Mareel M: Critical role of N-cadherin in myofibroblast invasion and migration in vitro stimulated by colon-cancer-cell-derived TGF-beta or wounding. J Cell Sci 117: 4691-4703, 2004

26. Bharti K, Nguyen MT, Skuntz S, Bertuzzi S and Arnheiter H: The other pigment cell: specification and development of the pigmented epithelium of the vertebrate eye. Pigment Cell Res 19: 380-394, 2006.

27. Strauss O: The retinal pigment epithelium in visual function. Physiol Rev 85: 845-881, 2005.

28. Winkler JL, Kedees MH, Guz Y and Teitelman G: Inhibition of connective tissue growth factor by small interfering ribonucleic acid prevents increase in extracellular matrix molecules in a rodent model of diabetic retinopathy. Mol Vis 18: 874-886, 2012.

29. Austin BA, Liu B, Li Z and Nussenblatt RB: Biologically active fibronectin fragments stimulate release of MCP-1 and catabolic cytokines from murine retinal pigment epithelium. Invest Ophthalmol Vis Sci 50: 2896-2902, 2009. 
30. Kothary PC, Badhwar J, Weng C and Del Monte MA: Impaired intracellular signaling may allow upregulation of CTGF-synthesis and secondary peri-retinal fibrosis in human retinal pigment epithelial cells from patients with age-related macular degeneration. Adv Exp Med Biol 664: 419-428, 2010.

31. Tikellis C, Cooper ME, Twigg SM, Burns WC and Tolcos M: Connective tissue growth factor is upregulated in the diabetic retina: Amelioration by angiotensin-converting enzyme inhibition. Endocrinology 145: 860-866, 2004.

32. Kuiper EJ, Witmer AN, Klaassen I, Oliver N, Goldschmeding R and Schlingemann RO: Differential expression of connective tissue growth factor in microglia and pericytes in the human diabetic retina. Br J Ophthalmol 88: 1082-1087, 2004

33. Cherian S, Roy S, Pinheiro A and Roy S: Tight glycemic control regulates fibronectin expression and basement membrane thickening in retinal and glomerular capillaries of diabetic rats. Invest Ophthalmol Vis Sci 50: 943-949, 2009.

34. Roy S, Cagliero E and Lorenzi M: Fibronectin overexpression in retinal microvessels of patients with diabetes. Invest Ophthalmol Vis Sci 37: 258-266, 1996.

35. Hughes JM, Kuiper EJ, Klaassen I, Canning P, Stitt AW, Van Bezu J, Schalkwijk CG, Van Noorden CJ and Schlingemann RO Advanced glycation end products cause increased $\mathrm{CCN}$ family and extracellular matrix gene expression in the diabetic rodent retina. Diabetologia 50: 1089-1098, 2007.

36. Martinez G and de Iongh RU: The lens epithelium in ocular health and disease. Int J Biochem Cell Biol 42: 1945-1963, 2010.

37. Neuzillet C, Tijeras-Raballand A, de Mestier L, Cros J, Faivre S and Raymond E: MEK in cancer and cancer therapy. Pharmacol Ther 141: 160-171, 2014.

38. Yuan L, Hu J, Luo Y, Liu Q, Li T, Parish CR, Freeman C, Zhu X, Ma W, Hu X, et al: Upregulation of heparanase in high-glucose-treated endothelial cells promotes endothelial cell migration and proliferation and correlates with Akt and extracellular-signal-regulated kinase phosphorylation. Mol Vis 18 1684-1695, 2012.

39. Qin D, Zheng XX and Jiang YR: Apelin-13 induces proliferation, migration, and collagen I mRNA expression in human RPE cells via PI3K/Akt and MEK/Erk signaling pathways. Mol Vis 19: 2227-2236, 2013.
40. Sasore T, Reynolds AL and Kennedy BN: Targeting the PI3K-Akt-mTOR pathway in ocular neovascularization. Adv Exp Med Biol 801: 805-811, 2014.

41. Yuan Z, Feng W, Hong J, Zheng Q, Shuai J and Ge Y: p38MAPK and ERK promote nitric oxide production in cultured human retinal pigmented epithelial cells induced by high concentration glucose. Nitric Oxide 20: 9-15, 2009.

42. Radeke MJ, Radeke CM, Shih YH, Hu J, Bok D, Johnson LV and Coffey PJ: Restoration of mesenchymal retinal pigmented epithelial cells by TGF $\beta$ pathway inhibitors: Implications for age-related macular degeneration. Genome Med 7: 58, 2015.

43. Simó R, Villarroel M, Corraliza L, Hernández C and Garcia-Ramírez M: The retinal pigment epithelium: Something more than a constituent of the blood-retinal barrier - implications for the pathogenesis of diabetic retinopathy. J Biomed Biotechnol 2010: 190724, 2010.

44. Dahrouj M, Desjardins DM, Liu Y, Crosson CE and Ablonczy Z: Receptor mediated disruption of retinal pigment epithelium function in acute glycated-albumin exposure. Exp Eye Res 137: 50-56, 2015.

45. Cao Y, Feng B, Chen S, Chu Y and Chakrabarti S: Mechanisms of endothelial to mesenchymal transition in the retina in diabetes. Invest Ophthalmol Vis Sci 55: 7321-7331, 2014

46. Sonnylal S, Xu S, Jones H, Tam A, Sreeram VR, Ponticos M, Norman J, Agrawal P, Abraham D and de Crombrugghe B: Connective tissue growth factor causes EMT-like cell fate changes in vivo and in vitro. J Cell Sci 126: 2164-2175, 2013.

47. Yang Z, Sun L, Nie H, Liu H, Liu G and Guan G: Connective tissue growth factor induces tubular epithelial to mesenchymal transition through the activation of canonical Wnt signaling in vitro. Ren Fail 37: 129-135, 2015.

48. Qiao M, Sheng S and Pardee AB: Metastasis and AKT activation. Cell Cycle 7: 2991-2996, 2008 